\title{
5) Carotid Baroreflex and alfa-receptors in SHR
}

T. Sasaki, N. Kajiwara, K. Kuroda, K. Sakai, H. Kanmatsuse, F. Sugiura, T. Okazaki, K. Tsujikawa, U. Nagano, M. Sugimori and S. Yabuki. Department of Cardiology, Surugadai Nihon University Hospital, Tokyo, 101 Japan.

There are many factors which regulate systemic hemodynamics, and autonomic nervous system has been proposed as one of the important factors among them. Many reflex systems have been shown to cause excitation of peripheral autonomic nervous system. Carotid baroreflex has been shown to participate the regulatory function of systemic circulation and alpha and beta receptors are shown to play an important role in carotid baroreflex.

The purpose of the experiment was designed to investigate the function of alpha 2 receptor in the systemic hemodynamics and its pathogenic role in SHR. Materials and methods: Male SHR and normotensive Wistar rats(WR) weighing 180 to $240 \mathrm{~g}$ were used. Anesthesia was performed by intraperitoneal urethane 50 to $100 \mathrm{mg} / 100 \mathrm{~g} \mathrm{B.W.} \mathrm{and} \mathrm{intravenous} \mathrm{alpha-chlorarose} 5 \mathrm{mg} / 100 \mathrm{~g} \mathrm{B.W.}$

Right carotid artery was cannulated and the cannula was connected to the pressure transducer for continuous recording of mean arterial pressure(MAP). Carotid baroreflex was conducted by occlusion of the carotid artery (co) of the other side. MAP was recorded before and after $\mathrm{CO}$, and effects of pretreatment with phenoxybenzamine(POB) and/or trazoline (TR) induced changes in MAP were assessed.

Results: Co induced biphasic response in MAP. After only pretreatment with large dose of TR (100mg), Co caused significant elevation of MAP as compared with that of control in SHR. After pretreatment with POB, CO caused decrease in MAP, however, TR pretreatment in addition with $P O B$, CO caused increase in MAP. The ratio of change in MAP after pretreatment with POB and TR was significantly greater in WR than that in SHR.

Discussion and conclusion: It has been suggested that the stimulation of alpha 2 receptor could inhihit catecholamine release presynapticall and the function of alpha 2 receptor might be impaired in SHR. The results of the present study that the pressor response to $C 0$ with pretreatment by TR was greater than that to without pretreatment in SHR and the pressorresponse to CO with pretreatment by $P O B$ and $T R$ was greater than that to $C O$ with pretreatment by $P O B$ alone in WR seem to support this hypothesis. We therefore conclude that alpha 2 receptor function may be impaired in SHR. 